\title{
Hemangioma cavernoso: relato de caso
}

\author{
Eduardo Queirós Miranda', José Edison da Silva Cavalcante², \\ Zacarias Cali ${ }^{3}$, Giordano Queirós Miranda ${ }^{1}$
}

Hospital Santa Mônica, Goiânia, GO, Brasil.

\section{RESUMO}

O hemangioma cavernoso é uma neoformação vascular benigna de origem congênita, caracterizando pela formação de grandes canais e espaços vasculares preenchidos com sangue. $O$ caso relatado no estudo chama atenção não só devido à magnitude da tumoração e ao comprometimento vascular, mas também pelo tempo de permanência da lesão, desde os 10 anos de idade. O tratamento para essa lesão varia com o tamanho, risco de ruptura do hemangioma e com a sintomatologia do paciente. As condutas que podem ser utilizadas são: ressecção cirúrgica; embolização arterial; enucleação cirúrgica e radioterapia. A raridade e complexidade do caso, somados à dificuldade de tratamento encontrada pela paciente no decorrer desses 20 anos de evolução tumoral, foram fatores que corroboraram para a apresentação desse caso.

\section{PALAVRAS-CHAVE}

Hemangioma, hemangioma cavernoso, neoplasias.

\section{ABSTRACT}

Cavernous hemangioma: case report

The cavernous hemangioma is a benign vascular neoformation of congenital origin, characterizing by the formation of large vascular spaces filled with blood. The case reported in this study differentiates itself from other cases of hemangiomas, not only because of the magnitude of the tumor and the vascular compromise, but also due to the time of permanence that this tumor was lodged since she was 10 . The treatment for this type of injury varies with the size and risk of a rupture of the hemangioma, and with the symptoms of the patient. Surgical resection, artery embolization, surgical enucleation and radiotherapy can be chosen by the doctor as alternatives for the treatment. The rarity and complexity of this case, added to the difficulty of treatment found by the patient during those 20 years of tumor development, compose the factors that corroborated to the presentation of this case.

\section{KEYWORDS}

Hemangioma, cavernous hemangioma, neoplasms.

1 Neurocirurgião do Hospital Santa Mônica, Goiânia, GO, Brasil.

2 Neurocirurgião, chefe do Serviço de Residência de Neurocirurgia do Hospital Santa Mônica, Goiânia, GO, Brasil.

3 Cirurgião pediátrico da Femina Maternidade, Goiânia, GO, Brasil. 


\section{Introdução}

Hemangiomas cavernosos são proliferações benignas congênitas dos vasos sanguíneos, sendo diferenciados dos demais hemangiomas pela formação de grandes canais e espaços vasculares com sangue. ${ }^{1}$

Definidos histologicamente como massas bem delimitadas, sem cápsula, e formados por grandes espaços vasculares cavernosos preenchidos de sangue, parcial ou completamente, e separados por uma escassa quantidade de tecido conjuntivo. ${ }^{2}$

Os angiomas cavernosos são geralmente oligossintomáticos, mas podem causar déficit neurológico súbito de maior gravidade pela ocorrência de trombose intravascular ou ruptura de canais levando a sangramentos intralesionais. ${ }^{3}$

A patogênese desses tumores é incerta, uma vez que exames complementares não mostram relação entre o hemangioma e a circulação patológica. ${ }^{1}$ Assim, é importante ressaltar que os angiomas cavernosos não são diagnosticados pela tomografia, e o exame ideal para sua detecção é a ressonância magnética de crânio. ${ }^{4}$

O caso citado visa mostrar a magnitude da tumoração, o comprometimento vascular e o tempo de permanência da lesão sem tratamento (Figura 1).

\section{Relato de caso}

Paciente, sexo feminino, 30 anos de idade, parda, com tumoração em região frontotemporal direita (Figuras 1,2 e 3) desde os 10 anos de idade.

Relatou início do tratamento desde os 10 anos, com realização de angiografia cerebral digital e embolização de vasos relacionados à tumoração, que nos últimos cinco anos cresceu progressivamente atingindo cerca de 12 centímetros.

Há três meses realizou outras embolizações em ramos da artéria carótida externa.

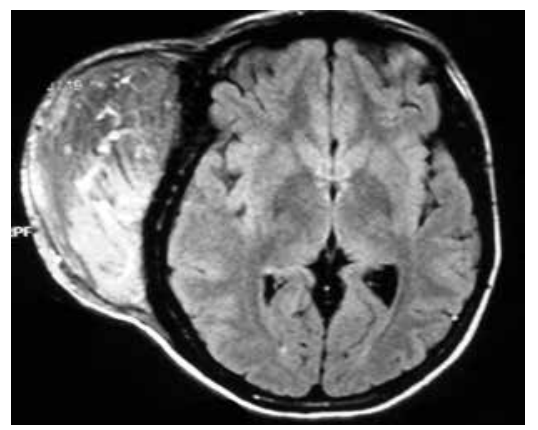

Figura 1 - Ressonância magnética de crânio com corte axial demonstrando tumoração em região frontoparietal direita.

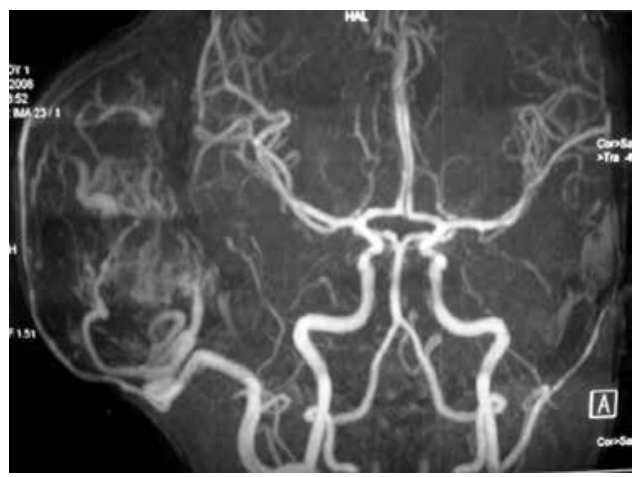

Figura 2 - Angiografia cerebral digital demonstrando intensa vascularização do tumor.

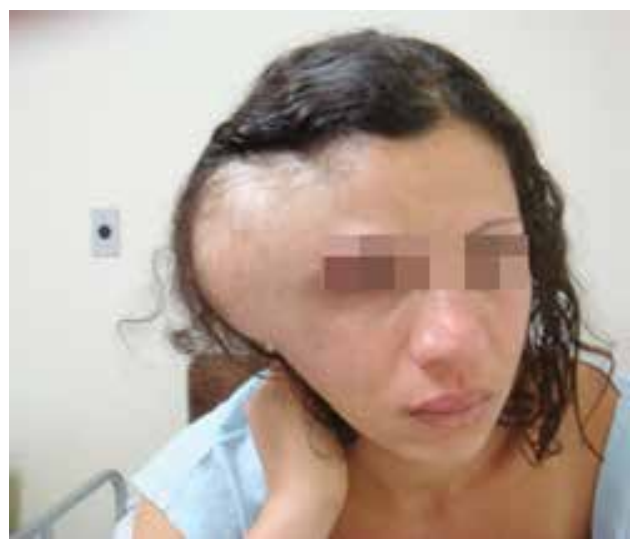

Figura 3 - Pré-operatório.

\section{Discussão}

Hemangioma é uma neoformação vascular benigna, de origem congênita, ${ }^{5}$ que se caracteriza pela proliferação de vasos sanguíneos, sendo mais comum na pele e em mucosas. Apesar de estudos em 1.308 lesões demonstrarem que a região de cabeça e pescoço é acometida em $56 \%$ dos casos, os hemangiomas podem localizar-se também em diferentes regiões e órgãos, como em ossos, músculos, sistema nervoso central e intestino grosso. Essa anomalia é mais comum no nascimento e na infância, podendo ocorrer também em adultos. ${ }^{5}$

Os hemangiomas são divididos, histologicamente, dependendo do tamanho microscópico dos vasos em: cavernosos e capilares. ${ }^{1}$ A forma cavernosa é menos frequente; são maiores, menos circunscritos e usualmente envolvem estruturas profundas, situação na qual são localmente destrutivos, não costumando regredir. É representado como uma massa esponjosa vermelho-azulada, formada por grandes espaços vasculares cavernosos preenchidos por sangue e separados por uma pequena quantidade de tecido conjuntivo. 
O quadro clínico dependerá da localização e do tamanho da lesão. ${ }^{1}$ Para os grandes hemangiomas com risco de ruptura ou para pacientes sintomáticos, as condutas utilizadas são ressecção cirúrgica (Figuras 4, 5 e 6), embolização arterial, enucleação cirúrgica e radioterapia. A cirurgia (Figuras 4 e 5), no entanto, deve ser indicada como última opção em circunstâncias de lesões sangrantes ou crescimento progressivo. ${ }^{6} \mathrm{~A}$ embolização prévia pode auxiliar no controle intraoperatório nas ressecções em locais de difícil acesso ou extensas. Já a radioterapia é, inicialmente, contraindicada para tratamentos de tumores na infância, devido seus efeitos colaterais.

Relatamos o caso de uma mulher de 30 anos com uma massa palpável em região temporal direita, com diagnóstico de hemangioma cavernoso extra-axial confirmado pelo exame histopatológico. Apesar de observarmos na literatura que a maioria dos casos de hemangioma cavernoso é assintomática e com possibilidades de involução, o quadro clínico apresentou-se com alguns sintomas e uma evolução do tamanho da lesão incomum para esse tipo de lesão, justificando a abordagem terapêutica (Figuras 4 e 5).

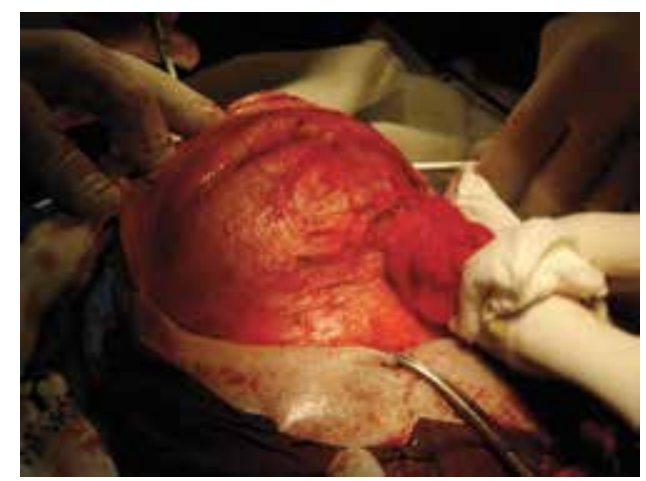

Figura 4 - Ressecção cirúrgica de hemangioma cavernoso extra-axial.

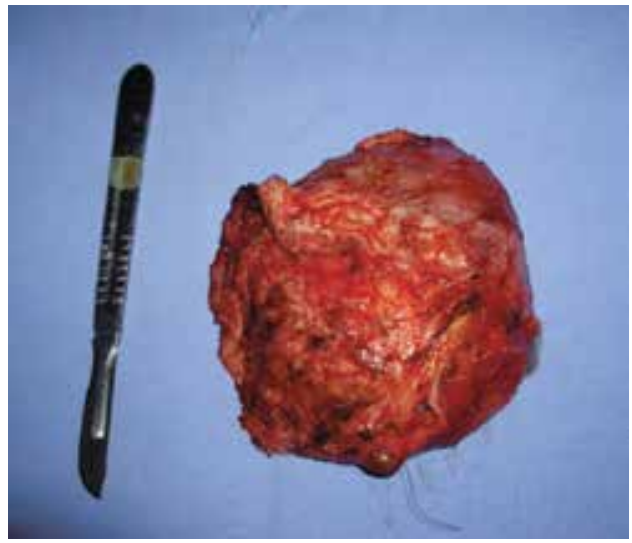

Figura 5 - Tumor após a ressecção cirúrgica.
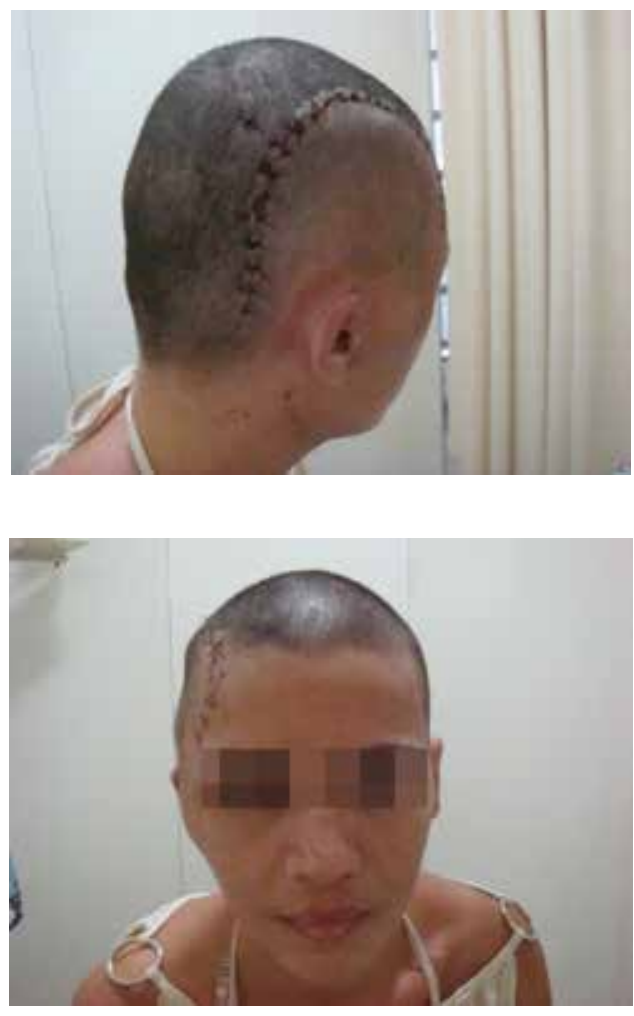

Figura 6 - Pós-operatório (10 dias).

\section{Considerações finais}

A neoformação vascular congênita relatada é um caso raro em que a paciente apresentava uma lesão tumoral extensa, sintomática, desde os 10 anos de idade, diferente da maioria dos casos publicados pela literatura.

$\mathrm{Na}$ tentativa de diminuir a irrigação da lesão, foram realizadas três sessões de embolização. A última tentativa, através da artéria carótida externa, que emitia ramos para o tumor, conseguiu amenizar o processo de vascularização e permitir a ressecção cirúrgica com maior segurança. A cirurgia foi realizada no Hospital Santa Mônica, Goiânia, GO. A paciente (Figura 6) encontra-se assintomática e sem lesões neurológicas evidentes.

Casos de hemangioma cavernoso extra-axial que atinge o músculo temporal, como no presente estudo, são raros. Isso mostra a complexidade do quadro relatado, desafiando inicialmente o tratamento para alguns cirurgiões.

\section{Conflito de interesses}

Os autores declaram inexistência de conflito de interesses na realização deste trabalho. 


\section{Referências}

1. Alcantara MP, Lazarini PR, Veiga JC, Barboza ES, Lancellotti $\mathrm{CL}$. Cerebellopontine angle cavernous hemangioma. Rev Bras Otorrinolaringol. 2006;72(6):850.

2. Silva FM, Andrade FB, Figueiredo PJ. Hemangioma. Unimep. 2000;12(1/2):57-9.

3. Luis MT, Rosa JS, Tâmega IE. Hemangioma cavernoso em pediatria. Rev Fac Ciênc Méd Sorocaba. 2004;6(1):34-7.

4. Marambaia PP, Santos OM, Gomes AM, Melo TF, Francisco TR, Araújo AH. Hemangioma cavernoso de seio maxilar. Relato de caso. Arq Int Otorrinolaringol. 2007;11(4):485-7.
5. Pedreira AV, Silva Cl, Freitas FJ, Barbosa VA, Machado MA Jr. [Intramedullary cavernous angioma of the spinal cord: case report]. Arq Neuropsiquiatr. 2004;62(2A):360-2 .

6. Gordon CR, Crockard HA, Symon L. Surgical management of spinal cord cavernoma. Br J Neurosurg. 1995;9(4):459-64.

\section{Endereço para correspondência}

Eduardo Queirós Miranda

Rua do Bordalo, quadra 58, lote 5

Privê Atlântico

74343-110 - Goiânia, GO, Brasil

Telefone: (62) 8286-9000

E-mail: eqm81@yahoo.com.br 